\title{
La ecología política urbana: veinte años de crítica, autocrítica y ampliación de fronteras en el estudio del metabolismo urbano*
}

\author{
Rubén Alejandro Villar Navascués \\ Universidad de Alicante. Instituto Interuniversitario de Geografía \\ rvnavascues@ua.es
}

\section{Resumen}

La ecología política urbana (EPU) es una disciplina que explora las interacciones entre factores sociales, políticos, económicos y naturales en la producción y en la reproducción de los entornos urbanos, que son entendidos como híbridos socionaturales. El objetivo de este artículo es, a partir de una revisión bibliográfica de la producción científica, identificar las principales temáticas y líneas de investigación para poner de relieve nuevos enfoques desde los que abordar críticamente las interacciones socioecológicas en las ciudades para poder plantear nuevos modelos urbanos sostenibles, tanto desde el punto de vista ecológico como social.

Palabras clave: ecología política urbana; análisis bibliográfico; metabolismo urbano; socionaturalezas; riesgos naturales

Resum. L'ecologia politica urbana: vint anys de critica, autocritica i expansió de fronteres en l'estudi del metabolisme urbà

L'ecologia política urbana (EPU) és una disciplina que explora les interaccions entre els factors socials, polítics, econòmics i naturals en la producció i en la reproducció d'ambients urbans, que són entesos com a híbrids socionaturals. L'objectiu d'aquest article és, a partir d'una revisió bibliogràfica de la producció científica, identificar les temàtiques i les línies de recerca principals per posar en relleu nous enfocaments que ajudin a fer front críticament a les interaccions socioecològiques que tenen lloc a les ciutats per poder plantejar models urbans sostenibles nous, tant des del punt de vista ecològic com social.

Paraules clau: ecologia política urbana; anàlisi bibliogràfica; metabolisme urbà; socionatures; riscos naturals

* Este artículo es resultado de la concesión de una beca predoctoral de Formación del Profesorado Universitario del Programa Nacional de Investigación Científica, Desarrollo e Innovación Tecnológica (FPU15/01144) y se inserta en el proyecto de investigación «Usos y gestión de recursos hídricos no convencionales en el litoral de las regiones de Valencia y Murcia como estrategia de adaptación a la sequía» (CSO2015-65182-CS-2-P), financiado por el Ministerio de Economía y Competitividad. 
Résumé. L'écologie politique urbaine : vingt ans de critique, d'autocritique et d'expansion des frontières dans l'étude du métabolisme urbain

L'écologie politique urbaine (EPU) est une discipline qui explore les interactions entre les facteurs sociaux, politiques, économiques et naturels dans la production et la reproduction des milieux urbains, interprétés comme hybrides socio-naturels. Le but de cet article est, à partir d'une revue bibliographique de la production scientifique, d'identifier les thèmes principaux et les axes de recherche pour mettre en évidence de nouvelles approches à partir desquelles aborder de manière critique les interactions socio-écologiques dans les villes pour être en mesure d'envisager de nouveaux modèles urbains durables, tant du point de vue écologique que social.

Mots-clés: écologie politique urbaine; analyse bibliographique; métabolisme urbain; socionatures; risques naturels

Abstract. Urban political ecology: Twenty years of criticism, self-criticism and expansion of frontiers in the study of urban metabolism

Urban political ecology (UPE) is a discipline that explores the interactions between social, political, economic and natural factors in the production and reproduction of urban environments, which are understood as socio-natural hybrids. Drawing from the literature on UPE, the aim of this article is to identify the main topics and research areas with a view to highlighting new approaches for critically addressing socio-ecological interactions in cities and proposing new urban models that are both ecologically and socially sustainable.

Keywords: Urban political ecology; literature review; urban metabolism; socio-natural; natural hazards

\section{Sumario}
1. Introducción
4. Conclusiones
2. Metodología
Referencias bibliográficas

3. Categorías de estudio en ecología política urbana

\section{Introducción}

Han pasado casi veinte años desde que el término ecología política urbana (EPU) fuera acuñado por Erik Swyngedouw en su obra The City as a hybrid: On nature, society and cyborg urbanization. Esta disciplina surge entonces como resultado de la falta de atención hacia temáticas urbanas por parte de la ecología política, así como por «la naturaleza abiertamente sociológica de gran parte de la teoría urbana del siglo XX» (Heynen et al., 2006a: 2), que no ha tenido en cuenta los procesos físicos ni ecológicos.

La EPU surge para aportar nuevos objetos de investigación y enfoques que complementan a los fundamentos teóricos clásicos de la ecología política. Entre 
ellos, encontramos un nuevo enfoque ontológico desde el que se replantea la noción de naturaleza, asumiendo que es un significante vacío y difícil de delimitar (Swyngedouw, 2011). Para evitar que su falta de concreción y sus significados múltiples sean utilizados con determinadas motivaciones políticas, la EPU propone abandonar el uso del concepto de naturaleza (Swyngedouw, 2007). En su lugar, los estudiosos de esta disciplina abogan por incorporar los planteamientos teóricos posthumanistas de autores como Latour (1993) o Haraway (1991) para concebir a las ciudades como naturalezas producidas socialmente que conforman híbridos socionaturales, mostrando, asimismo, la influencia que en ellas ejercen las relaciones de poder, que se dan bajo unas estructuras políticas y socioeconómicas determinadas.

Otra contribución esencial de la EPU es su particular interpretación del concepto metabolismo urbano. Este concepto ha sido utilizado por distintas disciplinas - entre ellas, encontramos la ecología industrial o la ecología urbana- como metáfora para explicar los procesos de intercambio por los cuales las ciudades transforman materias primas, energía y agua en ambiente construido, biomasa urbana y residuos (Decker et al., 2000).

En cambio, la EPU lo utiliza como aproximación epistemológica para interpretar el proceso de urbanización como un conjunto de acciones sociales y ecológicas que interaccionan metabolizando el ambiente «natural» a través de flujos de materiales de todo tipo, para producir esas segundas naturalezas o híbridos socionaturales (Castán-Broto et al., 2012; Heynen et al., 2006a; Zimmer, 2010). A diferencia de otras disciplinas, la EPU entiende que el metabolismo urbano tiene un fuerte componente político, ya que los flujos de materia se producen en base a unos procesos sociales determinados por el contexto histórico y las prácticas económicas favorecidas por los intereses de las élites en el poder. En definitiva, estos procesos metabólicos producen y reproducen materialmente la ciudad y convierten los elementos naturales en mercancías a partir de los llamados "vehículos metabólicos», entendidos como la red de infraestructuras que sostiene la circulación de los flujos metabólicos.

A partir de estas premisas, la producción científica de la EPU ha ido evolucionando a lo largo de sus casi dos décadas de existencia, distinguiendo dos generaciones de investigaciones (Heynen, 2014). En un primer momento, el lenguaje de la EPU fue principalmente marxista, aunque la influencia posthumanista y su carácter crítico han permitido la incorporación de nuevas aportaciones teóricas en la segunda generación de estudios. Por ejemplo, autores como Lawhon et al. (2014), Holifield (2009) o Gandy (2012) critican las limitaciones de la teoría marxista de la geografía urbana en la que se inserta gran parte de los estudios de la EPU y abogan por incluir influencias teóricas más heterogéneas, que pueden ser útiles para entender problemáticas ambientales locales. En concreto, Lawhon et al. (2014) exponen la necesidad de poseer un conocimiento más contextualizado de las relaciones socioecológicas urbanas, especialmente en las ciudades del sur global (Véron, 2010), realizando una reorientación epistemológica que incorpore, por ejemplo, el análisis de las prácticas cotidianas. Por otro lado, Holifield (2009) analiza la utilidad de la aplicación de la teoría del actor-red a los estudios de la EPU, al considerar el papel 
de los objetos «no humanos» en la generación de varias formas de injusticia y desigualdad como agentes activos de las relaciones urbanas (Gabriel, 2014).

Otra crítica que se ha hecho ha sido la preponderancia de los enfoques cualitativos y el escaso diálogo interdisciplinario por parte de la EPU (Angelo y Wachsmuth, 2014; Newell y Cousins, 2014). Ante esto, abogan por incorporar nuevas metodologías, procedentes de los diversos enfoques en torno al concepto de metabolismo urbano, para mejorar el entendimiento acerca de cómo funcionan las ciudades y para generar nuevas ideas y aplicaciones prácticas y normativas para la consecución de futuros urbanos sostenibles (Castán-Broto et al., 2012). Con respecto a esto, Newell y Cousins (2014) entienden que la metáfora del metabolismo urbano utilizada por la EPU está estancada metodológicamente, ya que hay una marcada ausencia del estudio de la ecología en las ciudades y del empleo de métodos cuantitativos. Por ello, plantean incorporar aspectos metodológicos utilizados por la ecología industrial, en concreto, el análisis del flujo de materia y el análisis del ciclo de vida, con los que se pueden planear y cuantificar las redes de flujos que circulan dentro y fuera de la ciudad, así como el estudio de las propiedades ecológicas y biofísicas del espacio urbano a partir de la teoría de la ecología urbana. Por último, se ha criticado que la EPU limite sus análisis a la ciudad, puesto que esta se convierte en el único espacio estudiado para unos procesos socionaturales de transformación que no están exclusivamente limitados a ella y se excluyen los vínculos espacio-temporales entre las ciudades y los lugares de producción y extracción de recursos que ayudan a sostenerlas (Angelo y Wachsmuth, 2014; Wachsmuth, 2012).

La realización y la aceptación de estas apreciaciones está permitiendo que evolucione la disciplina de la EPU, lo que posibilita que, a partir de un enfoque crítico que relaciona los aspectos ecológicos con los sociales, se enriquezca con distintas teorías, métodos y objetos de estudio y vaya más allá de la escala urbana en sus investigaciones (Arboleda, 2015; Gustafson et al., 2014). El objetivo de este artículo es revisar las principales temáticas y líneas de investigación que han sido tratadas por la EPU a nivel internacional para explorar hacia dónde evoluciona esta disciplina y qué alternativas y propuestas puede aportar para conseguir modelos urbanos más sostenibles. Esta aproximación bibliográfica supone una continuación del estudio desarrollado por Domene (2006), donde analizaba los orígenes, los objetivos y los principales estudios que hasta entonces había efectuado la EPU en el campo de la Geografía, principalmente. El artículo se estructura en tres partes. En primer lugar, se expondrá la metodología utilizada para el análisis de la producción bibliográfica de esta disciplina. Después, se realizará este análisis para cada una de las categorías de estudios identificadas, analizando la base teórica, así como las principales temáticas y líneas de investigación desarrolladas. Por último, se plantearán las conclusiones de este estudio.

\section{Metodología}

Para llevar a cabo esta aproximación bibliográfica, se ha efectuado una búsqueda de artículos, libros, capítulos de libros y comunicaciones en la base de 
datos SCOPUS, dada la prevalencia de las publicaciones de la EPU en lengua inglesa. La búsqueda se ha realizado mediante la selección de diferentes palabras para que actuaran como motor de localización. En algunos casos, se ha utilizado una combinación de varias palabras, dado el elevado número de publicaciones resultante de su búsqueda individual. Los términos, o las combinaciones entre ellos, utilizados para la selección bibliográfica, que aparecen en el título, en el resumen o en las palabras clave de las investigaciones, han sido: Urban Political Ecology, Urban Metabolism + Geography, Political Ecology + Socio Nature, Political Ecology + Flows + Urban. Asimismo, para complementar esta búsqueda con publicaciones efectuadas en lenguas latinas, se han consultado los términos ecología política urbana, metabolismo urbano y socionaturalezas en castellano, catalán, portugués y francés en otras bases de datos como el ISOC (CSIC) o el portal de difusión de producción científica DIALNET $^{1}$. No obstante, hay que señalar que este ejercicio supone tan solo una aproximación hacia las investigaciones más relevantes que tienen como referente teórico y temático a la EPU, quedándose fuera de él muchos estudios que se enmarcan en esta disciplina y, especialmente, aquellos que no han sido redactados en inglés o en castellano. En tercer lugar, tras comprobar que algunas investigaciones u obras relevantes para la EPU, como Social Power and the Urbanization of Water: Flows of Power, de Erik Swyngedouw, o City of Flows: Modernity, Nature and the City, de Maria Kaika, no fueron localizadas a través de la búsqueda en bases de datos, se han incluido algunas de las obras citadas en los diversos artículos que han analizado la producción científica más relevante de la disciplina (Zimmer, 2010; Swyngedouw y Kaika, 2014; Angelo y Wachsmuth, 2014; Heynen, 2014; Keil, 2003, 2005).

Del total de documentos recabado, 1.030 para las consultas en bases de datos, se realizó una primera selección en la que se escogieron aquellos textos que citaban explícitamente, en su título, en sus palabras clave o en su resumen, alguno de los siguientes términos: urban political ecology, urban metabolism, socionature o political ecology + urbanization. Posteriormente, se procedió a excluir aquellos artículos que compartían algunos de estos términos, pero que pertenecían a otras disciplinas como, por ejemplo, la ecología industrial. Por último, a partir de los resultados extraídos de esta selección, se procedió a realizar una agrupación de los temas principales de estudio en cuatro categorías: metabolismo hídrico, producción de socionaturalezas urbanas, flujos metabólicos y ecología política urbana de los riesgos naturales. Esta categorización se complementa con una serie de subdivisiones con el objeto de diferenciar las diversas temáticas contempladas en cada categoría (tabla 1). Gran parte de los estudios consultados presentan enfoques amplios acerca de los temas tratados que les permitirían estar presentes en varias de las temáticas diferenciadas, por lo que se han incluido en el aspecto que más representa a cada investiga-

1. Dado el escaso número de publicaciones encontradas a través de estos buscadores, se han incluido algunas publicaciones tras la consulta en línea de las revistas científicas Biblio $3 W$, Espaces et societés, Ecología Política, GEOUSP, L'Espace géographique, Scripta Nova y VertigO. 
Tabla 1. Estructura temática del estudio bibliográfico

\begin{tabular}{|c|c|}
\hline 1. Metabolismo hídrico & 3. Flujos metabólicos \\
\hline Urbanización, segregación socioespacial & Flujos de residuos. \\
\hline y acceso desigual al agua. & Flujos de contaminación. \\
\hline Cambios en la gobernanza urbana del agua. & Flujos alimentarios. \\
\hline $\begin{array}{l}\text { Ecología política urbana de la infraestructura } \\
\text { hídrica. }\end{array}$ & Otros flujos metabólicos. \\
\hline 2. Producción de socionaturalezas urbanas & 4. EPU de los riesgos naturales \\
\hline $\begin{array}{l}\text { Jardines privados urbanos. } \\
\text { Espacios verdes urbanos. }\end{array}$ & $\begin{array}{l}\text { Naturaleza política del discurso en torno } \\
\text { a la escasez hídrica. }\end{array}$ \\
\hline Ecología política de la infraestructura urbana. & $\begin{array}{l}\text { Estudios sobre construcción social de } \\
\text { vulnerabilidad. }\end{array}$ \\
\hline
\end{tabular}

Fuente: elaboración propia.

ción, evitando así encontrar una misma investigación en distintas categorías o temáticas.

\section{Categorías de estudio en ecología política urbana}

\subsection{Metabolismo hídrico}

Esta categoría engloba a los estudios sobre la circulación de los flujos de agua en las ciudades y sus implicaciones socioecológicas. Las primeras investigaciones en el campo de la EPU se enmarcaron en el ámbito del estudio del metabolismo hídrico urbano, como ejemplo paradigmático que permite entender la naturaleza política del agua a partir de las interacciones entre los factores humanos y los flujos naturales. En definitiva, se trata del análisis contextualizado de los flujos de agua en un marco territorial determinado, analizando de forma interrelacionada los sistemas socioeconómico, geográfico-ambiental y tecnoinstitucional.

Además, estos estudios revisan el tradicional concepto de ciclo hidrológico, adaptándolo al marco teórico de la EPU bajo el nombre de ciclo hidrosocial para remarcar la influencia antrópica que lo convierte en un proceso híbrido socionatural en el que agua y sociedad se influyen mutuamente en el espacio y a lo largo del tiempo (Swyngedouw, 2004, 2009; Linton y Budds, 2014; Schmidt, 2014). Por esta razón, en los estudios sobre metabolismo hídrico, se examinan los cambios en la gobernanza urbana del agua, ya que permite entender las tendencias existentes en cuanto a las relaciones de poder en torno a los recursos hídricos. Esta cuestión, además, es contextualizada en análisis teóricos más amplios que sitúan los procesos de privatización en la agenda de las políticas promovidas por la corriente neoliberal en su estrategia de «acumulación por desposesión» (Harvey, 2003; Swyngedouw, 2004, 2005). Todas las temáticas y líneas de investigación tratadas por los estudios sobre metabolismo hídrico están recogidas en la tabla 2. 
Tabla 2. Síntesis de las temáticas y líneas de investigación de los estudios sobre metabolismo hídrico

\begin{tabular}{|c|c|}
\hline Temática & Líneas de investigación \\
\hline \multirow{4}{*}{$\begin{array}{l}\text { Urbanización, segregación } \\
\text { socioespacial y acceso } \\
\text { desigual al agua. }\end{array}$} & Luchas sociales por el control del agua. \\
\hline & Desigualdad en el acceso al agua. \\
\hline & Servicios de saneamiento y asentamientos precarios. \\
\hline & Prácticas cotidianas alternativas de acceso al agua. \\
\hline \multirow[t]{2}{*}{$\begin{array}{l}\text { Cambios en la gobernanza } \\
\text { urbana del agua. }\end{array}$} & $\begin{array}{l}\text { Reformas neoliberales de la gestión hídrica y consecuencias } \\
\text { sobre el acceso al agua. }\end{array}$ \\
\hline & $\begin{array}{l}\text { Control de los flujos hídricos y reproducción de las estructuras } \\
\text { de poder. }\end{array}$ \\
\hline \multirow{4}{*}{$\begin{array}{l}\text { Ecología política urbana } \\
\text { de la infraestructura hídrica. }\end{array}$} & Estudios históricos de infraestructura hídrica. \\
\hline & $\begin{array}{l}\text { Infraestructura como reproductora de relaciones de poder } \\
\text { y desigualdades sociales. }\end{array}$ \\
\hline & Metabolismo hídrico y sector energético. \\
\hline & Flujos hídricos y conflictos bélicos. \\
\hline
\end{tabular}

Fuente: elaboración propia.

La primera temática analiza las relaciones entre el proceso de urbanización, la segregación socioespacial y el acceso desigual al agua. En esta materia, fue pionero Erik Swyngedouw, quien, en sus investigaciones sobre Guayaquil (1997, 2004), sentó las bases teóricas y metodológicas de gran parte de los análisis sobre metabolismo hídrico. En este sentido, se han desarrollado otros estudios en ciudades latinoamericanas, como en México DF (Castro, 2004). Gran parte de estos estudios se centran en las ciudades del sur global, donde la segregación socioespacial y las relaciones desiguales de poder resultan más evidentes, puesto que provocan intensas luchas sociales por el control y el acceso al agua. A pesar de estas premisas, Ranganathan y Balazs (2015), en su estudio sobre la periferia urbana de Exeter (EE. UU.) y Bangalore (India), encuentran similitudes entre los problemas de acceso al agua en ambas ciudades, lo cual desmonta la narrativa ambiental que vincula estos problemas exclusivamente con el Tercer Mundo. Desde la perspectiva de la ecología política feminista, Truelove (2011) estudia el acceso desigual al agua en Delhi (India) a través de las prácticas diarias guiadas por la distinción de género. Zimmer (2009, 2012, 2015) y Zimmer y Sakdapolrak (2012), por su parte, analizan los problemas relacionados con la falta de saneamiento en los asentamientos informales en la ciudad de Delhi, lo cual provoca innumerables problemas de higiene y favorece la aparición de enfermedades, como exponen Saravanan (2013) y Saravanan et al. (2014) para el caso de la ciudad india de Ahmedabad. Esta desigualdad en el acceso al agua determina que gran parte de la población tenga que conseguirla por medios informales o a través de prácticas cotidianas alternativas (Bakker, 2003). Estas estrategias de suministro ofrecen formas variadas, como ejemplifica el estudio de los llamados «regalos de agua» o transferencias de agua 
no remuneradas entre vecinos en la zona periurbana de Jartum (Blanchon y Graefe, 2012; Zug y Graefe, 2014).

La segunda temática gira en torno a los cambios en la gobernanza urbana del agua, y, más en concreto, a la privatización del suministro y de la gestión municipal, que suelen comportar la subida de tarifas y la exclusión del acceso al agua a los habitantes con menor nivel de renta, como muestran los ejemplos de distintas ciudades sudafricanas (Keil y Debbané, 2005; Loftus, 2006; Smith y Ruiters, 2006; Smith, 2001), Londres (Castro et al., 2003), Lima (Ioris, 2012a) o Guangzhou (Wehrhahn et al., 2013). Una segunda línea de investigación en esta temática se centra en el estudio del control de los flujos hídricos como estrategia para reproducir las estructuras de poder. Por ejemplo, Ioris (2012b) estudia los intereses de clase que dominan la gestión del agua en Baixada Fluminense (Brasil), que conducen a la reproducción de las desigualdades sociales y políticas. Masjuan et al. (2008) se centran en el agua como elemento de poder y en el conflicto político entre clases sociales imbricado en el desarrollo de la ciudad de Barcelona a lo largo del siglo xx. En la actualidad, las tendencias en torno a la evolución de la gobernanza del agua convergen en ciudades donde históricamente la participación del sector público y el sector privado en la gestión hídrica han sido muy diferentes, como es el caso de Madrid y Barcelona (March, 2013; March y Saurí, 2010). Estas luchas por el control del agua se ven reflejadas en los procesos de privatización de empresas y organismos públicos de gestión hídrica (March y Saurí, 2013).

La última temática analiza el papel y la importancia de las infraestructuras hídricas en el metabolismo de las ciudades, como pone de relieve Oliver (2006) en su estudio histórico sobre las obras de ingeniería del Támesis en Londres a finales del siglo XviII, que interrumpieron los flujos naturales del río y posibilitaron la explotación del mundo natural. Otra línea de investigación se centra en el papel de las infraestructuras como construcciones socioecológicas cruciales, no solo para sostener la circulación metabólica, sino también para reproducir las relaciones de poder y las desigualdades sociales (Gandy, 2004). Esta línea es ampliamente desarrollada por Erik Swyngedouw (1999, 2007, 2014, 2015), que reconstruye la historia del paisaje hidrosocial en España a lo largo del siglo xx, especialmente durante la dictadura franquista. A través de la construcción de embalses y gracias a los nuevos acuerdos geopolíticos que aseguraron los flujos de capitales, se cimentaron las ideas políticas del régimen en torno a la unidad nacional y se ganó el apoyo de las élites y de grupos sociales que se vieron beneficiados por la particular configuración de las políticas hídricas. En la investigación de Gandy (2008) sobre la ciudad india de Mumbai se analiza la debilidad de su infraestructura hídrica, explicada por sus orígenes coloniales o la naturaleza episódica del monzón, y cómo la creciente polarización social y las políticas clasistas profundizan en las desigualdades sociales heredadas. Domènech et al. (2013) ponen en evidencia las relaciones multiescalares de los conflictos socioecológicos movilizados por infraestructuras hídricas de gran envergadura, articulando la respuesta social desde la esfera urbana y rural por la desigual distribución de costes y beneficios 
ante un proyecto financiado por inversores internacionales. En otros casos, se analizan las relaciones entre metabolismo hídrico y sector energético, como ocurre en el estudio elaborado por Delgado-Ramos (2015), donde se exploran las repercusiones socioecológicas del nexo entre agua y energía para el caso de Ciudad de México. Cousins y Newell (2015) ahondan en esta línea de investigación combinando la teoría de la EPU con técnicas cuantitativas procedentes de la ecología industrial para analizar el metabolismo hídrico de Los Ángeles, poniendo de relieve los costes energéticos de las infraestructuras hídricas que movilizan los flujos hídricos hacia California.

La última línea de investigación en esta tercera temática analiza la importancia de las infraestructuras hídricas durante los conflictos bélicos, como sostenedoras de los flujos hídricos en las grandes ciudades. Graham (2006), a partir de su estudio sobre las nuevas estrategias bélicas implementadas en ciudades de Iraq, Kosovo o Palestina, basadas en la inutilización de las infraestructuras básicas como el suministro de agua y de electricidad, visibiliza el poder coercitivo de la demodernización forzada. Esta estrategia difiere de la utilizada por Franco durante la Guerra Civil española para apoderarse de Madrid, ya que centró sus bombardeos en los barrios obreros, mientras que los trabajadores del Canal de Isabel II consiguieron evitar la interrupción del suministro de agua durante casi tres años, lo cual permitió afrontar el esfuerzo bélico con alguna posibilidad de éxito (Gorostiza y Saurí, 2013; Gorostiza et al., 2014).

\subsection{Producción de socionaturalezas urbanas}

Las socionaturalezas urbanas son el resultado espacial de las interacciones entre los procesos sociales, económicos y políticos con los flujos metabólicos. Las ciudades, entendidas como híbridos, constituyen en sí mismas un producto socionatural, pero en su interior cristalizan otra serie de naturalezas urbanas que expresan las desiguales relaciones de poder y las estructuras socioeconómicas dominantes. Esta categoría pretende diferenciar la colección de socionaturalezas urbanas que han sido analizadas por parte de la EPU y los problemas socioecológicos que generan, por lo que muchos casos de estudio complementan sus análisis con enfoques de justicia ambiental. Las temáticas y las líneas de investigación tratadas en esta categoría están recogidas en la tabla 3.

La primera temática hace referencia al estudio de los jardines privados urbanos, considerados «espacios de consumo y de producción que actúan como representaciones de aspectos sociales y culturales más amplios» (Domene, 2006: 174). El desarrollo de modelos urbanos dispersos ha traído consigo la importación de la tipología de jardín atlántico norteamericano, cuyo componente principal es el césped. Las repercusiones socioambientales de la producción de estos jardines ha motivado que Robbins (2007) y otros autores (Robbins et al., 2001; Robbins y Sharp, 2003, 2006, 2008; Robbins y Birkenholtz, 2003) hayan analizado el riesgo ambiental que supone la utilización de una gran cantidad de productos químicos para mantener este césped residencial, así como los procesos económicos que promueven su implantación. 
Tabla 3. Síntesis de las temáticas y de las líneas de investigación de los estudios sobre producción de socionaturalezas urbanas

\begin{tabular}{ll}
\hline Temática & Líneas de investigación \\
\hline Jardines privados urbanos. & $\begin{array}{l}\text { Repercusiones socioecológicas de la producción de jardines } \\
\text { privados urbanos. } \\
\text { Tendencias en el consumo de agua doméstica derivada } \\
\text { de la expansión de jardines privados urbanos. }\end{array}$ \\
\hline Espacios verdes urbanos. & $\begin{array}{l}\text { Distribución y producción desigual de los espacios verdes } \\
\text { urbanos. }\end{array}$ \\
& $\begin{array}{l}\text { Reformas neoliberales y espacios verdes urbanos. } \\
\text { Huertos urbanos como espacios de justicia socioecológica. }\end{array}$ \\
\hline Ecología política de & $\begin{array}{l}\text { Socionaturalezas industriales: acumulación de capital } \\
\text { la infraestructura urbana. }\end{array}$ \\
& y gentrificación ecológica. \\
& Socionaturalezas postindustriales. \\
& Infraestructura urbana y expansión de la periferia ecológica. \\
& Nuevos modelos de ciudad y acumulación de capital. \\
& Políticas, sostenibilidad y desigualdad social. \\
\hline
\end{tabular}

Fuente: elaboración propia.

Además, se pone de relieve la contradicción entre el nivel cultural de los propietarios de los jardines, en su mayoría concienciados por el equilibrio ecológico, y el uso indiscriminado que se hace de estos productos químicos. Dicha antítesis es analizada por Kaika (2004), al estudiar la evolución históricogeográfica del hogar burgués occidental.

La repercusión sobre el consumo de agua que generan estos jardines se ha analizado en la costa mediterránea española, en territorios expuestos a graves déficits hídricos, especialmente en las provincias de Barcelona y Girona (Domene y Saurí, 2003, 2006; Garcia et al., 2013a, 2013b, 2013c, 2014; Padullés et al., 2014a, 2014b; Parés, 2005; Parés et al., 2004). La elección de tipologías de jardín atlántico con jardín con césped y piscina en el área metropolitana de Barcelona ha sido relacionada con aspectos sociales, como el nivel de renta y su consideración como «bien posicional» (Domene, 2014; Parés et al., 2013), lo que resulta en una diferenciación de las naturalezas producidas según el nivel de ingresos o la clase social, y en que más de la mitad del consumo doméstico durante el verano y un tercio del anual sea destinado a riego (Domene et al., 2005). En la provincia de Alicante, por el contrario, se ha relacionado la existencia de otro tipo de socionaturalezas urbanas - como los jardines de tipo mediterráneo y las plantas ornamentales - con el elevado precio del agua (Morote y Hernández, 2014; Hernández et al., 2014).

Otra temática de estudios trata sobre los espacios verdes urbanos, entendidos como productos socioecológicos, cuya distribución espacial desigual responde a los mismos procesos socioeconómicos y políticos que afectan al resto de espacios de las ciudades y que, en definitiva, conforman distintas realidades 
ambientales que afectan a la calidad de vida de sus habitantes. Mientras la mayor parte del arbolado urbano se localiza en propiedades privadas, según el nivel de renta, la mayoría de la población es dependiente de la inversión pública en espacios verdes, la cual, en muchos casos, no se produce, lo que favorece esta distribución desigual (Birge-Liberman, 2010; Heynen, 2006a; Heynen y Perkins, 2005; Heynen et al., 2006b; Kitchen, 2013; Perkins et al., 2004). Además, la privatización de los terrenos sobre los que se asientan los bosques urbanos erosiona aún más la equidad social en las ciudades. En sus estudios sobre la privatización parcial de la Reserva Forestal de Karura, en Nairobi, Njeru (2010, 2013) muestra cómo las presiones ejercidas por los inversores occidentales sobre el régimen de Moi para llevar a cabo este tipo de reformas neoliberales provocaron la caída de este régimen.

Las investigaciones acerca de los huertos urbanos aparecen como otra línea de estudio, entendidos como híbridos socionaturales que desempeñan un papel importante como espacios verdes donde construir la soberanía alimentaria, la justicia socioecológica en barrios desfavorecidos y el cambio sociopolítico, poniendo en práctica iniciativas sostenibles a escala local (Classens, 2015; Domene y Saurí, 2007; McLain et al., 2014; Milbourne, 2012; Moore, 2006; Shillington, 2013).

La tercera temática se centra en el papel de las infraestructuras urbanas, entendidas como sistemas sociotécnicos que permiten la circulación metabólica de los recursos naturales, siendo por ello elementos clave en la comprensión de los problemas y de las dinámicas socioecológicas (Monstadt, 2009). Algunos análisis se centran en el papel de las infraestructuras como vehículos metabólicos o socionaturalezas industriales que facilitan y mejoran la circulación del capital (Cooke y Lewis, 2010). En este sentido, algunos estudios entienden las iniciativas de restauración y mejora de los frentes marítimos (Bunce y Desfor, 2007; Desfor y Vesalon, 2008; Hagerman, 2007) como generadoras de procesos de gentrificación ecológica que profundizan las desigualdades sociales como consecuencia del acceso mejorado a los bienes ambientales, que aumentan el precio del suelo y atraen a grupos sociales acomodados, desplazando a la población económicamente más vulnerable. Otras investigaciones sobre infraestructura urbana se centran en las repercusiones que el declive de la industria, y la consiguiente desaceleración del metabolismo urbano, han traído a la vida cotidiana de los residentes, analizando las socionaturalezas postindustriales en Detroit (Millington, 2013) o en la periferia de Glasgow (Ioris, 2014).

Otro análisis en torno a las infraestructuras urbanas estudia el papel que estas desempeñan en la expansión de la periferia ecológica, tanto para la extracción de recursos (Arboleda, 2015) como para la eliminación de residuos (Crivello, 2015), resultando una redistribución física de los flujos de materia que es acompañada por un cambio en el tipo de organización social, lo cual comporta una gobernanza urbana de las infraestructuras donde el poder local está subyugado a los intereses de las empresas multinacionales. A este respecto, Monstadt (2009) argumenta la dificultad de llevar a cabo cambios tecnológicos radicales, ya que son los regímenes sociotecnológicos, entendidos como confi- 
guraciones relativamente estables de instituciones técnicas e infraestructuras, los que guían los procesos de variación tecnológica. Por ello, los nuevos modelos urbanos de ecociudad y smart city, donde se plantean innovaciones técnicas y de gobernanza de la infraestructura urbana, se enmarcan en los mismos regímenes sociotécnicos actuales, cuya voluntad política neoliberal está lejos de diseñar modelos de ciudad orientados hacia la participación política, la inclusión social o la sostenibilidad ecológica (Domínguez y Fogué, 2013). En este sentido, los nuevos modelos de ciudad surgidos en torno al concepto de desarrollo sostenible se han promovido bajo enfoques tecnocráticos de mercado que buscan «reverdecer» y reinventar el capitalismo (Davidson y Gleeson, 2014; Kaika y Swyngedouw, 2012; Kenis y Lievens, 2015). La promoción de estos modelos urbanos, que suponen un nuevo nicho de mercado para la acumulación de capital, se fundamentan a partir del discurso de la crisis climática y de una noción despolitizada de la sostenibilidad, que enmascara los intereses económicos detrás de las soluciones tecnológicas y la transferencia de atribuciones y de dinero público al ámbito privado. Esto, además, reproduce las desigualdades socioecológicas y las estructuras de poder (Bulkeley et al., 2014; Castán-Broto y Bulkeley, 2013; Caprotti, 2014; Cidell, 2009; Kear, 2007; Keil y Boudreau, 2006; March y Ribera-Fumaz, 2014a, 2014b). Por dicho motivo se critica que las cuestiones sociales son escasamente desarrolladas en las estrategias de planificación sostenible (Davidson y Arman, 2014) y en la política urbana (Ellis, 2011; Troy, 2014), donde es común que, a partir de premisas como la sostenibilidad, se produzcan procesos de gentrificación ecológica (March y Ribera-Fumaz, 2014a; Quastel et al., 2012) y de exclusión social (Safransky, 2014).

\subsection{Flujos metabólicos}

El metabolismo urbano moviliza distintos tipos de flujos de materia que, alrededor del mundo, vinculan los sistemas de ciudades en forma de red. La EPU centra una parte relevante de sus investigaciones en desentrañar cómo se produce la circulación de estos distintos tipos de mercancías y qué repercusiones socioecológicas provocan dichos flujos. Se identifica, además, que la probabilidad de conflicto socioecológico crece cuando estos flujos son aumentados, interrumpidos o redirigidos, como resultado de la configuración de las relaciones de poder desiguales, bajo regímenes de gobernanza específicos, y de la influencia que ejerce la infraestructura urbana, entendida como vehículo a través del cual circulan estos flujos distribuidos de forma dispar.

Por ello, aunque es común vincular las investigaciones de la EPU con cuestiones de justicia ambiental (JA), esta categoría de estudios está especialmente ligada a dicha disciplina, ya que incide en los conflictos sociales que se crean fruto de la circulación desigual de los flujos metabólicos. La EPU contribuye a ampliar el marco teórico de la JA y a superar su orientación simplemente empírica reconociendo explícitamente que las ecologías urbanas desiguales se producen bajo la influencia de la estructura socioeconómica capitalista, inci- 
Tabla 4. Síntesis de las temáticas y de las líneas de investigación de los estudios sobre flujos metabólicos

\begin{tabular}{ll}
\hline Temática & Líneas de investigación \\
\hline Flujos de residuos. & Residuos sólidos urbanos y recicladores informales. \\
& $\begin{array}{l}\text { Distribución socioespacial desigual de los residuos urbanos } \\
\text { y justicia ambiental. } \\
\text { Prácticas cotidianas y flujos de residuos. }\end{array}$ \\
\hline Flujos de contaminación. & EPU de la contaminación del suelo. \\
& EPU de la contaminación doméstica. \\
& EPU de la contaminación atmosférica. \\
\hline Flujos alimentarios. & Flujos alimentarios y capitalismo. \\
& Nuevos paisajes alimentarios urbanos. \\
& Biografía del flujo de alimentos. \\
& Procesos de gentrificación ecológica asociados a los flujos \\
alimentarios.
\end{tabular}

Fuente: elaboración propia.

diendo en que los conflictos socioecológicos locales pueden prestarse a generalizaciones que los inscriban en escalas más amplias (Heynen et al., 2006b; Ostos, 2014). Asimismo, Perkins (2007) y Holifield (2009) proponen la inclusión de la teoría del actor-red (TAR) como un enfoque alternativo crítico que incide en la influencia que los llamados «objetos no humanos" tienen en la producción de desigualdades, lo que puede resultar útil para los estudios de la EPU que traten temáticas relacionadas con la JA para abordar cuestiones que enfoques eminentemente marxistas no podrían plantear. Todas las temáticas y líneas de investigación asociadas a los estudios sobre flujos metabólicos han sido sintetizadas en la tabla 4.

Una primera temática está centrada en el análisis de los flujos de residuos, entendidos como el resultado final de la circulación metabólica, que provocan conflictos socioecológicos y reflejan las desigualdades socioeconómicas estructurales (Solíz, 2014). Los conflictos en las ciudades de países del sur global en torno a la acumulación desproporcionada de residuos, una deficiente gestión municipal y el desarrollo de un sector informal de reciclaje de residuos es una línea de investigación prolífica en la EPU (Bjerkli, 2015; Leonard, 2012; Parizeau, 2015; Yates y Gutberlet, 2011). Estos estudios analizan las problemáticas sanitarias y las desigualdades socioecológicas y espaciales derivadas de la circulación de estos flujos y la respuesta por parte del sector informal del reciclaje, lo que supone una adaptación a las socionaturalezas urbanas existentes ante la incapacidad del sistema de ofrecer una alternativa estructural en la gestión de residuos. Otros estudios analizan las problemáticas derivadas de iniciativas públicas destinadas a gestionar estos residuos, como la introducción 
de las plantas incineradoras en Delhi, que provocó una respuesta conjunta de ciudadanos de clase media, preocupados por la contaminación atmosférica, junto con trabajadores del sector informal de residuos que veían amenazada su fuente de subsistencia (Demaria y Schindler, 2015). En torno a esta temática, también se desarrollan otras líneas de investigación, como las relaciones entre diversos conflictos acerca de la distribución espacial desigual de los residuos urbanos y la justicia ambiental (Njeru, 2006; Moore, 2008) o la relación de los flujos de residuos con las prácticas cotidianas en referencia a las mascotas en el espacio público urbano (Instone y Sweeney, 2014).

Una segunda temática estudia los flujos de contaminación en el espacio urbano. Desde la perspectiva de la geografía física crítica, McClintock (2015) analiza la deposición de plomo antropogénico en los suelos urbanos, entendidos como construcciones híbridas socionaturales, lo que permite situar el origen de este flujo de contaminación en el contexto histórico, político y económico en el que fue producido, para denunciar el desigual impacto espacial y étnico de las concentraciones de plomo. Una segunda línea de estudio se centra en la EPU de los flujos domésticos de contaminación, analizando el legado tóxico del asbesto en las ciudades australianas, así como la influencia de las prácticas cotidianas y de la distinción de clase y de género en el desigual grado de exposición (Houston y Ruming, 2014). Biehler (2009), por su parte, estudia la exposición a pesticidas tóxicos, asociada a la gestión de plagas, en las viviendas sociales públicas en EE. UU. a lo largo del siglo Xx. Una última línea de investigación en torno a esta temática está constituida por los flujos de la contaminación atmosférica (Bruzzelli, 2008; Graham, 2015; Kay et al., 2015; Véron, 2006). A partir de una amplia gama de metodologías, estas investigaciones analizan las implicaciones de la gobernanza y las políticas ambientales sobre la desigual repercusión de la contaminación atmosférica en los distintos grupos sociales, entre otros aspectos.

La tercera temática se centra en los flujos alimentarios producidos en el marco de una economía global. Desde una perspectiva teórica, Marvin y Medd (2006) analizan cómo, a través de redes tanto locales como globales de producción alimentaria, circula el flujo de grasa que produce la obesidad en las ciudades norteamericanas, interconectando los metabolismos de las poblaciones, los cuerpos y las infraestructuras. Heynen (2006b) se centra en los conflictos urbanos alimentarios en Milwaukee, en lo que ha calificado como ecología política del hambre urbana, poniendo de relieve la influencia de la desigualdad estructural capitalista y los procesos políticos en la producción social de la desigualdad urbana. A este respecto, han surgido los nuevos paisajes alimentarios de las ciudades, como los huertos urbanos analizados en el apartado anterior, asociados a las cuestiones de malnutrición, hambre y obesidad y de desigualdad socioespacial, a través del movimiento por la justicia alimentaria urbana, que busca conectar formas más democráticas de planeamiento con un nuevo flujo de alimentos (Morgan, 2015). Saguin (2014) examina la complejidad de estos flujos a través de un enfoque de la biografía del flujo de alimentos, en este caso analizando las prácticas materiales cotidianas asociadas con la producción 
acuícola de pescado en Filipinas, que transforma los flujos tradicionales, los procesos metabólicos y las fronteras socioecológicas urbanas para asegurar el suministro de alimentos. Los flujos alimentarios también se relacionan con ciertas dinámicas y procesos de gentrificación ecológica, a partir de la aparición de establecimientos de comida orgánica cuyos consumidores suelen pertenecer a clases sociales acomodadas (Moragues-Faus y Morgan, 2015). La aparición de estos establecimientos se relaciona con incipientes incrementos del valor de las propiedades, lo cual provoca un desplazamiento de la población más vulnerable económicamente. Anguelovski (2015) explora las relaciones entre la justica alimentaria urbana y los procesos de gentrificación ecológica en Jamaica Plain, un barrio de Boston donde la variedad de opciones alimentarias sensible a la diversidad cultural de sus habitantes es amenazada por la apertura de una tienda de alimentos orgánicos en lugar de un supermercado de productos latinos, lo cual crea un sentimiento de desplazamiento para los habitantes de dicho origen paralelo a la desaparición de opciones económicas de provisión de alimentos.

Por último, hay que mencionar una serie de estudios que analizan aspectos muy dispares, comprendidos en la temática titulada «Otros flujos metabólicos». En primer lugar, destaca el estudio de Lawhon (2013), que se centra en los flujos y en la metabolización del alcohol en Ciudad del Cabo a través de las prácticas cotidianas y de los circuitos informales que motivan la identificación de las personas que los movilizan como infraestructuras que aseguran el suministro en los bares ilegales. En segundo lugar, los estudios ubicados en la ecología política de la salud urbana, que vinculan la epidemiología ecosocial con la EPU, como el análisis desarrollado por Mulligan et al. (2012), estudian las relaciones entre los flujos de enfermedades, como el dengue, con factores políticos e institucionales, como la falta de atención a cuestiones de salud pública por parte del planeamiento en la ciudad emergente de Putraya (Malasia).

\subsection{Ecología politica urbana de los riesgos naturales}

La dimensión social de los riesgos naturales, entendidos como la conjunción de un peligro natural con la vulnerabilidad de una sociedad expuesta a ese peligro, permite abordar distintos casos de estudio explicando cómo la vulnerabilidad es socialmente construida. La aplicación del marco teórico de la EPU enlaza con este tema, al desentrañar las causas socioeconómicas estructurales y las relaciones desiguales de poder que conducen a la injusticia ambiental en las ciudades, lo cual genera situaciones de vulnerabilidad para una parte de la población y, por tanto, de riesgo natural. Una aproximación a los estudios y a las temáticas que, en relación con los riesgos naturales, lleva a cabo la EPU está sintetizada en las líneas de investigación que aparecen en la tabla 5. 
Tabla 5. Síntesis de las temáticas y de las líneas de investigación de los estudios sobre ecología política urbana de los riesgos naturales

\begin{tabular}{ll}
\hline Temática & Líneas de investigación \\
\hline $\begin{array}{l}\text { Naturaleza política del discurso } \\
\text { en torno a los riesgos naturales. }\end{array}$ & Producción social de la sequía y la escasez hídrica. \\
\hline $\begin{array}{ll}\text { Estudios sobre construcción } \\
\text { social de vulnerabilidad. }\end{array}$ & $\begin{array}{l}\text { Desregulación ambiental y producción de vulnerabilidad. } \\
\text { Desarrollo desigual y producción de vulnerabilidad. }\end{array}$ \\
\hline
\end{tabular}

Fuente: elaboración propia.

Una primera temática trata de la naturaleza política del discurso en torno a los riesgos naturales, en particular con respecto a las sequías, que son entendidas como un producto socionatural. A través del discurso que considera la escasez hídrica como una consecuencia de la fatalidad natural o del cambio climático, se pretende que la población afectada no cuestione los mecanismos económicos, sociales y políticos que han conducido a la producción social de la sequía y que, en definitiva, sirve para beneficiar a las élites socioeconómicas a través de la imposición de soluciones técnicas y la exclusión a los habitantes de bajos ingresos del acceso al agua (Giglioli y Swyngedouw, 2008; Ioris, 2012c; Kaika, 2003, 2005, 2006; Otero et al., 2011).

Además, en torno a esta cuestión, la naturaleza lineal o insostenible del metabolismo urbano, que depende fuertemente de la extracción de recursos hídricos de una periferia cada vez más amplia, es presentada como una fuente particular de vulnerabilidad (Castán-Broto et al., 2012: 854). Marks (2015), por su parte, analiza la gobernanza urbana de los desastres naturales a partir del caso de estudio de las inundaciones de 2011 en Bangkok, considerando cómo el discurso gubernamental atribuye al cambio climático los resultados de estas inundaciones y no a las verdaderas causas que se identifican con los resultados desiguales del proceso de urbanización, los cambios en los usos del suelo que afectan al aumento de la escorrentía, una infraestructura anticuada y unas políticas concretas que protegieron a la ciudad central frente a las áreas periféricas.

Una segunda temática, que pone en contacto a la EPU con la JA, comprende los estudios que tratan acerca de la producción social de la vulnerabilidad y su repercusión en la creación o en la intensificación de los riesgos naturales. En primer lugar, encontramos una serie de investigaciones que tratan acerca de la desregulación ambiental y de la producción de vulnerabilidad. Esta relación es puesta de manifiesto por Baxter (2014), al analizar las interrelaciones entre la especulación inmobiliaria y las decisiones políticas que llevaron a ampliar la frontera urbana de Nueva Orleans hacia sus humedales del este, que fueron gravemente afectados tras el paso del huracán Katrina. Simon (2014), por su parte, analiza el incendio de 1991 en las colinas de Oakland, una interfaz urbano-forestal, a partir de un enfoque espacial-histórico. Este autor entiende la producción de la vulnerabilidad 
como un proceso relacional que se encuentra permanentemente en activo y que provoca el desencadenamiento de nuevas vulnerabilidades que, bajo la influencia de unas políticas neoliberales que redujeron los presupuestos para prevención y gestión de incendios, produjeron el mayor incendio hasta la fecha en California en términos de viviendas destruidas. Además, este mismo desastre natural sirve de vehículo para diferenciar entre las vulnerabilidades experimentadas (materiales) y las interpretadas (políticas), lo que puede reforzar la vulnerabilidad de una parte de la población urbana y exportarla a otras partes de la ciudad (Simon y Dooling, 2013). Gustafson (2015a, 2015b) estudia cómo, tras una serie de deslizamientos en un área suburbana de Carolina del Norte, se produjeron un conjunto de mapas sobre riesgo de deslizamiento que, bajo el enfoque de la TAR, son entendidos como objetos de conocimiento ambiental que podrían reducir la vulnerabilidad de los habitantes, al comprender estos su exposición a ese peligro natural. Pero esos mapas no fueron utilizados ni publicados, debido a los conflictos que provocaron entre algunos propietarios de la zona y la presión de la industria inmobiliaria, lo que provocó una falta de información y un paisaje de riesgo innecesario. El estudio de Collins (2010) se centra en la aplicación de los conceptos de marginación y facilitación en los estudios sobre producción de vulnerabilidad. Ambos resultan de utilidad para entender la producción de riesgos de inundación desiguales en El Paso y Ciudad Juárez, ya que incorporan el papel que desempeñan las instituciones gubernamentales y el mercado en la trasferencia de riesgos, proporcionando una base para entender la producción multiescalar del riesgo desigual. Mientras que los mecanismos institucionales deniegan una adecuada protección contra el riesgo de inundaciones al creciente número de trabajadores de las maquiladoras en la frontera norte de México, en El Paso la búsqueda de entornos ambientales atractivos ha motivado que las residencias de la élite se encuentren en zonas de riesgo de inundación.

Otra línea argumental incide en las consecuencias de los desarrollos urbanos desiguales en la producción de vulnerabilidad. El precoz estudio de Pelling (1999) analiza, a partir del análisis histórico-geográfico, por qué motivo alrededor del $90 \%$ de la población de Guyana se ve afectada por el riesgo de inundación. Para este autor, no es solo el sistema físico quien crea este riesgo, sino sobre todo los sistemas políticos, sociales y económicos, que desempeñan un papel crucial en la producción de vulnerabilidad. Los procesos de rápida urbanización en las grandes ciudades de los países en desarrollo y el acceso limitado a la vivienda han conducido a un crecimiento de barrios marginales y a un aumento de la vulnerabilidad ante las inundaciones, acentuada por la degradación medioambiental y la consiguiente merma de la resiliencia natural ante los desastres, como muestran los estudios sobre Lagos (Ajibade y McBean, 2014); Yakarta (Padawangi y Douglass, 2015); la conurbación de Rawalpindi-Islamabad (Mustafa, 2005); Bangalore (Ranganathan, 2015); o Santiago de Chile (Romero et al., 2010). 


\section{Conclusiones}

Esta aproximación bibliográfica permite comprobar cómo la EPU presenta actualmente una renovada vitalidad académica por la incorporación de nuevas metodologías, la mejora del diálogo interdisciplinario que permite ampliar el marco teórico o la ampliación de los casos de estudio más allá del ámbito físico urbano. Asimismo, es evidente la hegemonía de la literatura anglosajona en la EPU, que ha privilegiado los casos de estudio en países de habla inglesa. A pesar de esto, la producción de la EPU en América Latina ha sido importante, dado que las intensas desigualdades sociales y el alto grado de urbanización han provocado que los conflictos socioecológicos sean numerosos. En España, por otro lado, la producción científica de la EPU y los casos de estudio analizados se concentran especialmente en Cataluña, aunque la penetración de los conceptos provenientes de la EPU en análisis de geografía crítica y metabolismo hídrico en la península Ibérica está desarrollándose en otros contextos académicos, como Alicante, Sevilla o Madrid (Cabello et al., 2015; Del Moral, 2013; Larrabeiti, 2012; Hernández-Mora y Del Moral, 2015). Por tanto, a nivel nacional, uno de los retos de esta disciplina para la próxima década será mantener dicha tendencia ampliando los casos de investigación a otros ámbitos.

La importancia de la evolución que presenta la EPU radica en el desarrollo de nuevos enfoques que permitan mejorar el entendimiento de las interacciones socioecológicas en las ciudades. Este hecho, junto con la necesidad de abordar las problemáticas socioecológicas urbanas en nuevos contextos geográficos y el planteamiento de nuevas temáticas y líneas de investigación, permitirá, en última instancia, abordar nuevas propuestas políticas y mecanismos de respuesta ante la hegemonía de las políticas neoliberales a escala global, con el objetivo de plantear nuevos modelos urbanos que sean sostenibles socioecológicamente. Por ello, el desarrollo de las investigaciones en EPU debe aportar, y en muchos casos lo hace, algo más que el conocimiento y la crítica de un problema socioecológico específico, como la capacidad de concienciar sobre la naturaleza política de las interacciones socioecológicas. Esto hace necesario mejorar la comunicación de los resultados de estas investigaciones más allá de los ámbitos académicos, ya que la inclusión de las propuestas planteadas en un discurso contrahegemónico a las políticas neoliberales solo será posible si son apoyadas por amplios sectores de la población.

\section{Referencias bibliográficas}

Angelo, Hillary y Wachsmuth, David (2014). «Urbanizing Urban Political Ecology: A Critique of Methodological Cityism». International Journal of Urban and Regional Research [en línea], 39 (1), 16-27. <http://dx.doi.org/10.1111/1468-2427.12105>

Arboleda, Martín (2015). «In the Nature of the Non-City: Expanded Infrastructural Networks and the Political Ecology of Planetary Urbanisation». Antipode [en línea], 6 de agosto. $<$ http://dx.doi.org/ 10.1111/anti.12175> 
Cabello, Violeta; Willaarts, Barbara A.; Aguilar, Monica y Moral, Leandro del (2015). "River basins as social-ecological systems: Linking levels of societal and ecosystem water metabolism in a semiarid watershed». Ecology and Society [en línea], 20 (3), 20. <http://dx.doi.org/10.5751/ES-07778-200320>

Castán-Broto, Vanesa; Allen, A. y Rapoport, E. (2012). «Interdisciplinary Perspectives on Urban Metabolism». Journal of Industrial Ecology [en línea], 16, 851-861.

<http://dx.doi.org/ 10.1111/j.1530-9290.2012.00556.x>

Decker, E.H.; Elliott, S.; Smith, F.A.; Blake, D.R. y Rowland, F.S. (2000). «Energy and material flow through the urban ecosystem». Annual Review of Energy and the Environment, 25, 685-740.

Gabriel, Nate (2014). «Urban Political Ecology: Environmental Imaginary, Governance, and the Non-Human». Geography Compass [en línea], 8 (1), 38-48. <http://dx.doi.org/10.1111/gec3.12110>

Gandy, Matthew (2012). "Queer ecology: Nature, sexuality, and heterotopic alliances». Environment and Planning D: Society and Space [en línea], 30 (4), 727-747. <http://dx.doi.org/ 10.1068/d10511>

Gustafson, Seth; Heynen, Nik; Rice, Jennifer L.; Gragson, Ted; Shepherd, J. Marshall y Strother, Christopher (2014). «Megapolitan Political Ecology and Urban Metabolism in Southern Appalachia». Professional Geographer [en línea], 66 (4), 664-675.

<http://dx.doi.org/10.1080/00330124.2014.905158>

Haraway, Donna J. (1991). Simians, Cyborgs and Women: The Reinvention of Nature. Londres: Free Association Books.

Hernández-Mora, Nuria y Moral, Leandro del (2015). «Developing markets for water reallocation: Revisiting the experience of Spanish water mercantilización». Geoforum [en línea], 62, 143-155. <http://dx.doi.org/10.1016/j.geoforum.2015.04.011>

Heynen, Nik (2014). "Urban Political Ecology I: The Urban Century». Progress in Human Geography [en línea], 38 (4), 598-604. <http://dx.doi.org/10.1177/0309132513500443>

Heynen, Nik; Kaika, Maria y Swyngedouw, Erik (eds.) (2006a). In the Nature of Cities: Urban Political Ecology and the Politics of Urban Metabolism. Londres: Routledge.

Holifield, Ryan (2009). "Actor-Network theory as a critical approach to environmental justice: A case against synthesis with urban political ecology». Antipode [en línea], 41 (4), 637-658. <http://dx.doi.org/ 10.1111/j.1467-8330.2009.00692.x>

Kaika, Maria y Swyngedouw, Erik (2012). «Cities, natures and the political imaginary». Architectural Design [en línea], 82 (4), 22-27. <http://dx.doi.org/ 10.1002/ad.1423>

KeIL, Roger (2003). «Urban political ecology». Urban Geography [en línea], 24 (8), 723-738. <http://dx.doi.org/10.2747/0272-3638.24.8.723>

- (2005). «Progress report: Urban political ecology». Urban Geography [en línea], 26 (7), 640-651.

<http://dx.doi.org/10.2747/0272-3638.26.7.640> 
LARRABEITI, Juan J. (2012). «Producción de nuevas "naturalezas urbanas” y sus consecuencias sobre el consumo de agua en Alicante». Investigaciones Geográficas, 58, 143-170.

Latour, Bruno (1993). We Have Never Been Modern. London: Harvester Wheatsheaf.

LAwHon, Mary; ERnstson, Henrik y Silver, Jonathan (2014). «Provincializing urban political ecology: Towards a situated UPE through African urbanism». Antipode [en línea], 46 (2), 497-516. <http://dx.doi.org/10.1111/anti.12051>

Moral, Leandro del (2013). "Crisis del capitalismo global: Desarrollo y medio ambiente». Documents d'Anàlisi Geogràfica [en línea], 59 (1), 77-103. <http://dx.doi.org/10.5565/rev/dag.36>

Newell, Joshua P. y Cousins, Joshua J. (2014). «Boundaries of urban metabolism: Towards a political-industrial ecology». Prog. Hum. Geogr. [en línea]. <http://dx.doi.org/10.1177/ 0309132514558442>

Swyngedouw, Erik (1996). "The City as a hybrid: On nature, society and cyborg». Capitalism, Nature, Socialism. Urbanization, 7 (2), 65-80. <http://dx.doi.org/10.1080/10455759609358679>

- (2004). Social Power and the Urbanization of Water. Londres: Oxford University Press.

- (2006). "Circulations and Metabolisms: (Hybrid) Natures and (Cyborg) Cities». Science as Culture [en línea], 15 (2), 105-121.

<http://dx.doi.org/10.1080/09505430600707970>

- (2007). «Impossible/Undesirable Sustainability and the Post-Political Condition». En: Krueger, J.R. y Gibbs, D. (eds.). The Sustainable Development Paradox. Nueva York: Guilford, 13-40.

- (2011). «Nature does not exist!: Sustainability as Symptom of a Depoliticized Planning». Urban, 1, 41-66.

Swyngedouw, Erik y Heynen, Nik (2003). «Urban political ecology, justice, and the politics of scale». Antipode [en línea], 35 (5), 898-918. <http://dx.doi.org/10.1111/j.1467-8330.2003.00364.x>

Swyngedouw, Erik y KaIKa, Maria (2014). «Urban Political Ecology: Great Promises, Deadlock... and New Beginnings?». Documents d'Anàlisi Geogràfica [en línea], 60 (3), 459-481. <http://dx.doi.org/10.5565/rev/dag.155>

VÉron, René (2010). «Small cities, neoliberal and sustainable development in the global south: A conceptual framework and research agenda». Sustainability [en línea], 2 (9), 2822-2848. <http://dx.doi.org/10.3390/su2092833>

Wachsmuth, David (2012). "Three Ecologies: Urban Metabolism and the Society-Nature Opposition». Sociological Quarterly [en línea], 53 (4), 506-523. <http://dx.doi.org/ 10.1111/j.1533-8525.2012.01247.x>

Zimmer, Anna (2010). «Urban Political Ecology. Theoretical concepts, challenges, and suggested future directions». Erdkunde, 64 (4), 343-354. <http://dx.doi.org/10.3112/erdkunde.2010.04.04>

\section{Metabolismo hidrico}

BAKKer, Karen (2003). «Archipelagos and networks: Urbanization and water privatization in the South». The Geographical Journal [en línea], 169 (4), 328-341. <http://dx.doi.org/10.1111/j.0016-7398.2003.00097.x> 
- (2005). "Neoliberazing Nature?: Market Environmentalism in water Supply in England and Wales». Annals of the Association of American Geographers [en línea], 95 (3), 542-565.

<http://dx.doi.org/10.1111/j.1467-8306.2005.00474.x>

Blanchon, David y Graefe, Olivier (2012). «Radical Political Ecology and Water in Khartoum». L'Espace Géographique, 41, 35-50.

Castro, Jose E. (2004). "Urban water and the politics of citizenship: The case of Mexico City Metropolitan Area during the 1980s and 1990s». Environment and Planning A [en línea], 36 (2), 327-346.

<http://dx.doi.org/10.1068/a35159>

Castro, Jose E.; Kaika, Maria y Swyngedouw, Erik (2003). «London: Structural continuities and institutional change in water management». European Planning Studies [en línea], 11 (3), 283-298. <http://dx.doi.org/10.1080/09654310303635>

Cousins, Joshua J. y Newell, Joshua P. (2015). "A political-industrial ecology of water supply infrastructure for Los Angeles». Geoforum [en línea], 58, 38-50. <http://dx.doi.org/10.1016/j.geoforum.2014.10.011>

Delgado-Ramos, Gian C. (2015). "Water and the political ecology of urban metabolism: The case of Mexico City». Journal of Political Ecology, 22 (1), 98-114.

Domènech, Laia; Saurí, David; March, Hug (2013). «Contesting large-scale water supply projects at both ends of the pipe in Kathmandu and Melamchi Valleys, Nepal». Geoforum [en línea], 47, 22-31. <http://dx.doi.org/10.1016/j.geoforum.2013.02.002>

GANDY, Matthew (2004). «Rethinking urban metabolism: Water, space and the modern city». City [en línea], 8 (3), 363-379.

<http://dx.doi.org/10.1080/1360481042000313509>

- (2008). "Landscapes of disaster: Water, modernity, and urban fragmentation in Mumbai». Environment and Planning A [en línea], 40 (1), 108-130. <http://dx.doi.org/10.1068/a3994>

Gorostiza, Santiago; March, Hug; Saurí, David (2014). "Urban Ecology Under Fire": Water Supply in Madrid during the Spanish Civil War (1936-1939)». Antipode [en línea], 47 (2), 360-379.

<http://dx.doi.org/10.1111/anti.12111>

Gorostiza, Santiago y SAurí, David (2013). «Salvaguardar un recurso precioso: La gestión del agua en Madrid durante la guerra civil española (1936-1939)». Scripta Nova. Revista Electrónica de Geografía y Ciencias Sociales [en línea], XVII (457). Barcelona: Universidad de Barcelona, 20 de noviembre. <http://www.ub.es/ geocrit/sn/sn-457.htm>.

Graham, Stephen (2006). «Urban metabolism as target: Contemporary war as forced demodernization». En: Heynen, Nik; Kaika, Maria y Swyngedouw, Erik (eds.): In the nature of cities: Urban political ecology and the politics of urban metabolism. Londres: Routledge, 245-265.

<http://dx.doi.org/10.2747/0272-3638.28.2.206>

Harvey, David (2003). The New Imperialism. Oxford: Oxford University Press.

Ioris, Antonio A.R. (2012a). "The persistent water problems of Lima, Peru: Neoliberalism, institutional failures and social inequalities». Singapore Journal of Tropical Geography [en línea], 33 (3), 335-350.

<http://dx.doi.org/10.1111/sjtg.12001> 
- (2012b). "Applying the strategic-relational approach to urban political ecology: The water management problems of the Baixada Fluminense, Rio de Janeiro, Brazil». Antipode [en línea], 44 (1), 122-150. <http://dx.doi.org/10.1111/j.1467-8330.2011.00848.x>

Keil, Roger y Debbané, Anne M. (2005). «Scaling discourse analysis: Experiences from Hermanus, South Africa and Walvis Bay, Namibia». Journal of Environmental Policy and Planning, 7 (3), 257-276.

Linton, Jamie y BudDS, Jessica (2014). "The hydrosocial cycle: Defining and mobilizing a relational-dialectical approach to water». Geoforum [en línea], 57, 170-180. <http://dx.doi.org/10.1016/j.geoforum.2013.10.008>

LofTus, Alex (2006). «The metabolic processes of capital accumulation in Durban's waterscape». En: Heynen, Nik; Kaika, Maria y Swyngedouw, Erik (eds.). In the nature of cities: Urban political ecology and the politics of urban metabolism [en línea]. Londres: Routledge, 165-182. <http://dx.doi.org/10.2747/0272-3638.28.2.206>

- (2011). "Applied Urban Ecology: A Global Framework». En: Geographical Perspectives on a Radical Political Ecology of Water. University of London, 193-203.

MarCH, Hug (2013). "Taming, controlling and metabolizing flows: Water and the urbanization process of Barcelona and Madrid (1850-2012)». European Urban and Regional Studies [en línea], 22 (4), 350-367. <http://dx.doi.org/10.1177/0969776412474665>

March, Hug y Saurí, David (2010). «Flujos de agua, flujos de capital: Sistemas de abastecimiento y gobernanza del agua en Madrid y Barcelona». Investigaciones Geográficas [en línea], 51, 7-26. <http://dx.doi.org/10.14198/INGEO2010.51.01>

- (2013). «The Unintended Consequences of Ecological Modernization: Debt-induced Reconfiguration of the Water Cycle in Barcelona». Environment and Planning $A$ [en línea], 45 (9), 2064-2083. <http://dx.doi.org/10.1068/a45380>

Masjuan, Eduard; March, Hug; Domene, Elena y Saurí, David (2008). "Conflicts and Struggles over Urban Water Cycles: The case of Barcelona 1880-2004». Tijdschrift Voor Economische En Sociale Geografie [en línea], 99 (4), 426-439. <http://dx.doi.org/10.1111/j.1467-9663.2008.00477.x>

Oliver, Stuart (2006). "The desire to metabolize nature: Edward Loveden, William Vanderstegen, and the disciplining of the river Thames». En: Heynen, Nik; KaIKa, Maria y Swyngedouw, Erik (eds.). In the nature of cities: Urban political ecology and the politics of urban metabolism [en línea]. Londres: Routledge, 90-105. <http://dx.doi.org/10.2747/0272-3638.28.2.206>

Ranganathan, Malini y Balazs, Carolina (2015). "Water marginalization at the urban fringe: Environmental justice and urban political ecology across the NorthSouth divide». Urban Geography [en línea], 36 (3), 403-423. <http://dx.doi.org/10.1080/02723638.2015.1005414>

Saravanan, V.S. (2013). «Urbanizing diseases: Contested institutional terrain of water-and vector-borne diseases in Ahmedabad, India». Water International [en línea], 38 (7), 875-887. <http://dx.doi.org/10.1080/02508060.2013.851363>

Saravanan, V.S.; Mavalankar, Dileep; Kulkarni, Suhas P.; Nussbaum, Sven y Weigelt, Martin (2014). «Metabolized-Water Breeding Diseases in Urban India: 
Sociospatiality of Water Problems and Health Burden in Ahmedabad City». Journal of Industrial Ecology [en línea], 19 (1), 93-103.

<http://dx.doi.org/10.1111/jiec.12172>

Schmidt, J.J. (2014). «Historicising the hydrosocial cycle». Water Alternatives [en línea], 7 (1), 220-234.

Smith, Laila (2001). "The urban political ecology of water in Cape Town». Urban Forum [en línea], 12 (2), 204-224.

<http://dx.doi.org/10.1007/s12132-001-0016-4>

SMITH, Laila y RuITERS, Greg (2006). «The public/private conundrum of urban water: A view from South Africa». En: Heynen, Nik; KaIka, Maria y Swyngedouw, Erik (eds.): In the nature of cities: Urban political ecology and the politics of urban metabolism [en línea]. Londres: Routledge. 183-198. <http://dx.doi.org/10.2747/0272-3638.28.2.206>

Swyngedouw, Erik (1997). «Power, nature, and the city: The conquest of water and the political ecology of urbanization in Guayaquil, Ecuador: 1880-1990». Environment and Planning $A$ [en línea], 29 (2), 311-332. $<$ http://dx.doi.org/10.1068/a290311>

- (1999). «Modernity and Hybridity: Nature, Regeneracionismo, and the Production of the Spanish Waterscape, 1890-1930». Annals of the Association of American Geographers [en línea], 89 (3), 443-465. <http://dx.doi.org/10.1111/0004-5608.00157>

- (2004). Social Power and the Urbanization of Water: Flows of Power. Oxford: Oxford University Press.

- (2005). «Dispossessing $\mathrm{H}_{2} \mathrm{O}$ : The contested terrain of water privatization». Capitalism, Nature, Socialism [en línea], 16 (1), 81-98.

<http://dx.doi.org/10.1080/1045575052000335384>

- (2007). «Technonatural revolutions: The scalar politics of Franco's hydro-social dream for Spain, 1939-1975». Transactions of the Institute of British Geographers [en línea], 32 (1), 9-28.

<http://dx.doi.org/10.1111/j.1475-5661.2007.00233.x>

- (2009). "The political economy and political ecology of the hidrosocial cycle». Journal of Contemporary Water Research and Education [en línea], 142 (1), 56-60. <http://dx.doi.org/10.1111/j.1936-704x.2009.00054.x>

- (2014). "Not a drop of water...": State, modernity and the production of nature in Spain, 1898-2010». Environment and History [en línea], 20 (1), 67-92. <http://dx.doi.org/10.3197/096734014X13851121443445>

- (2015). Liquid Power: Contested Hydro-Modernities in Twentieth Century Spain [en línea]. Cambridge: MIT Press. <http://dx.doi.org/10.7551/mitpress/9780262029032.001.0001>

Truelove, Yaffa (2011). «(Re-)Conceptualizing water inequality in Delhi, India through a feminist political ecology framework». Geoforum [en línea], 42 (2), 143-152. <http://dx.doi.org/10.1016/j.geoforum.2011.01.004>

Wehrhahn, Rainer; Helten, M. y Bercht, Anna L. (2013). «Political ecology of potable water in Guangzhou: Actors and social vulnerabilities». Geographische Rundschau, 65 (10), 38-45.

Zimmer, Anna (2009). «Social relations in the waste waterscapes: The case of Delhi's informal settlements». UGEC Viewpoint, 9, 9-12.

- (2012). «Fragmented governance, divided cities. The need for an integrated view on urban waste water: A Case Study of Delhi». Dams, Rivers and People, 10 (2), 9-13. 
- (2015). "Urban Political Ecology in Megacities: The Case of Delhi's Waste Water». En: Singh, R.B. (ed.). Urban Development Challenges, Risks and Resilience in Asian Mega Cities [en línea]. Tokio: Springer. 119-139. <http://dx.doi.org/10.1007/978-4-431-55043-3_7>

Zimmer, Anna y Sakdapolrak, Patrick (2012). "The social practices of governing: Analysing waste water governance in a Delhi slum». Environment and Urbanization Asia [en línea], 3 (2), 325-341. <http://dx.doi.org/10.1177/0975425312473228>

Zug, Sebastian y GraEfe, Olivier (2014). «The gift of water. social redistribution of water among neighbours in Khartoum». Water Alternatives, 7 (1), 140-159.

\section{Producción de naturalezas urbanas}

Arboleda, Martín (2015). "In the Nature of the Non-City: Expanded Infrastructural Networks and the Political Ecology of Planetary Urbanisation». Antipode [en línea]. <http://dx.doi.org/10.1111/anti.12175>

Birge-Liberman, Phil (2010). "(Re)greening the city: Urban park restoration as a spatial fix». Geography Compass [en línea], 4 (9), 1392-1407. <http://dx.doi.org/10.1111/j.1749-8198.2010.00374.x>

Bulkeley, Harriet; Castán-Broto, Vanesa y MaAssen, Anne (2014). «Low-Carbon transitions and the reconfiguration of Urban Infraestructure». Urban Studies [en línea], 51 (7), 1471-1486. <http://dx.doi.org/10.1177/0042098013500089>

Bunce, Sussanah y Desfor, Gene (2007). "Introduction to "Political ecologies of urban waterfront transformations"». Cities [en línea], 24 (4), 251-258. <http://dx.doi.org/10.1016/j.cities.2007.02.001>

CAprotti, Federico (2014). «Eco-urbanism and the eco-city, or, denying the right to the city?». Antipode [en línea], 46 (5), 1285-1303. <http://dx.doi.org/10.1111/anti.12087>

Castán-Broto, Vanesa y Bulkeley, Harriet (2013). "Maintaining climate change experiments: Urban political ecology and the everyday reconfiguration of urban infrastructure». International Journal of Urban and Regional Research [en línea], 37 (6), 1934-1948. <http://dx.doi.org/10.1111/1468-2427.12050>

Cidell, Julie L. (2009). «A political ecology of the built environment: LEED certification for green buildings». Local Environment [en línea], 14 (7), 621-633. <http://dx.doi.org/10.1080/13549830903089275>

Classens, Michael (2015). «The nature of urban gardens: Toward a political ecology of urban agriculture». Agriculture and Human Values [en línea], 32 (2), 229-239. <http://dx.doi.org/10.1007/s10460-014-9540-4>

Cooke, Jason y LewIS, Robert (2010). «The nature of circulation: The urban political ecology of Chicago's Michigan avenue bridge, 1909-1930». Urban Geography [en línea], 31 (3), 348-368. <http://dx.doi.org/10.2747/0272-3638.31.3.348>

Crivello, Silvia (2015). "Political ecologies of a waste incinerator in Turin, Italy: Capital circulation and the production of urban natures». Cities [en línea], 48, 109-115.

<http://dx.doi.org/10.1016/j.cities.2015.06.010> 
Davidson, Kathryn y Arman, Michael (2014). «Planning for sustainability: An assessment of recent metropolitan planning strategies and urbanpolicy in Australia». Australian Planner [en línea], 51 (4), 296-306. <http://dx.doi.org/10.1080/07293682.2013.877508>

Davidson, Kathryn y GleEson, Brendan (2014). «The Sustainability of an Entrepreneurial City?». Internacional Planning Studies [en línea], 19 (2), 173-191. <http://dx.doi.org/10.1080/13563475.2014.880334>

Desfor, Gene y VesAlon, Lucian (2008). «Urban expansion and industrial nature: A political ecology of Toronto's port industrial district». International Journal of Urban and Regional Research [en línea], 32 (3), 586-603. <http://dx.doi.org/10.1111/j.1468-2427.2008.00806.x>

Domene, Elena (2006). «La ecología política urbana: Una disciplina emergente para el análisis del cambio socioambiental en entornos ciudadanos». Documents d'Anàlisi Geogràfica, 48, 167-178.

- (2014). «Cambio en los patrones del consumo de agua en la Barcelona suburbana: Estilos de vida y renta como factores explicativos». Investigaciones Geográficas [en línea], 61, 39-53. <http://dx.doi.org/10.14198/ingeo2014.61.03>

Domene, Elena y SAurí, David (2003). «Modelos urbanos y consumo de agua: El riego de jardines privados en la región metropolitana de Barcelona». Investigaciones Geográficas [en línea], 32, 5-17. <http://dx.doi.org/10.14198/INGEO2003.32.02>

- (2006). «Urbanization and water consumption: Influential factors in the metropolitan region of Barcelona». Urban Studies [en línea], 43 (1), 605-623. <http://dx.doi.org/10.1080/00420980600749969>

- (2007). «Urbanization and class-produced natures: Vegetable gardens in the Barcelona Metropolitan Region». Geoforum [en línea], 38 (2), 287-298. <http://dx.doi.org/10.1016/j.geoforum.2006.03.004>

Domene, Elena; Saurí, David; Parés, Marc (2005). «Urbanization and sustainable resource use: The case of garden watering in the metropolitan region of Barcelona». Urban Geography [en línea], 26 (6), 520-535. <http://dx.doi.org/10.2747/0272-3638.26.6.520>

Domínguez, Fernando y Fogué, Uriel (2013). «Technifying Public Space and Publicizing Infrastructures: Exploring New Urban Political Ecologies through the Square of General Vara del Rey». International Journal of Urban and Regional Research [en línea], 37 (3), 1035-1052. <http://dx.doi.org/10.1111/1468-2427.12052>

Ellis, Rowan (2011). «Who’s Participation? Who's Sustainability?: A Critical Analysis of Initiatives for Urban Sustainability in India». Scottish Geographical Journal [en línea], 127 (3), 193-208. <http://dx.doi.org/10.1080/14702541.2011.616863>

Garcia, Xavier; Llausàs, Albert y Ribas, Anna (2013a). «Landscaping patterns and sociodemographic profiles in suburban areas: Implications for water conservation along the Mediterranean coast». Urban Water Journal [en línea], 11 (1), 31-41. <http://dx.doi.org/10.1080/1573062x.2012.758296>

Garcia, Xavier; Muro, Melanie; Ribas, Anna; Llausàs, Albert; Jeffrey, Paul y Saurí, David (2013b). «Attitudes and behaviours towards water conservation on the Mediterranean coast: The role of sociodemographic and place-attachment factors». Water International [en línea], 38 (3), 283-296. <http://dx.doi.org/10.1080/02508060.2013.794641> 
Garcia, Xavier; Ribas, Anna; Llausàs, Albert y Saurí, David (2013c). «Socio-demographic profiles in suburban developments: Implications for water-related attitudes and behaviors along the Mediterranean coast». Applied Geography [en línea], 41, 46-54.

<http://dx.doi.org/10.1016/j.apgeog.2013.03.009>

Garcia, Xavier; Ribas, Anna y Llausàs, Albert (2014). "Jardines privados y consumo de agua en las periferias urbanas de la comarca de La Selva (Girona)». Investigaciones Geográficas [en línea], 61, 55-69.

<http://dx.doi.org/10.14198/ingeo2014.61.04>

HAGERMAN, Chris (2007). «Shaping neighborhoods and nature: Urban political ecologies of urban waterfront transformations in Portland, Oregon». Cities [en línea], 24 (4), 285-297. <http://dx.doi.org/10.1016/j.cities.2006.12.003>

Hernández, María; Morales, Alfredo y Saurí, David (2014). «Ornamental plants and the production of nature(s) in the Spanish real estate boom and bust: The case of Alicante». Urban Geography [en línea], 35(1), 71-85. <http://dx.doi.org/ 10.1080/02723638.2013.871813>

Heynen, Nik (2006a). «Green urban political ecologies: Toward a better understanding of inner-city environmental change». Environment and Planning $A$ [en línea], 38 (3), 499-516. <http://dx.doi.org/10.1068/a37365>

Heynen, Nik y Perkins, Harold A. (2005) «Scalar dialectics in green: Urban private property and the contradictions of the neoliberalization of nature». Capitalism, Nature, Socialism [en línea], 16 (1), 99-113. <http://dx.doi.org/10.1080/1045575052000335393>

Heynen, Nik; Perkins, Harold A. y Roy, Parama (2006b). «The political ecology of uneven urban green space: The impact of political economy on race and ethnicity in producing environmental inequality in Milwaukee». Urban Affairs Review [en línea], 42 (1), 3-25. <http://dx.doi.org/10.1177/1078087406290729>.

Ioris, Antonio A.R. (2014). «The Urban Political Ecology of Post-industrial Scottish Towns: Examining Greengairs and Ravenscraig». Urban Studies [en línea], 51(8), 1576-1592. <http://dx.doi.org/10.1177/0042098013497408>

KaIKA, Maria (2004). «Interrogating the Geographies of the Familiar: Domesticating Nature and Constructing the Autonomy of the modern home». International Journal of Urban and Regional Research [en línea], 28 (2), 265-286. <http://dx.doi.org/10.1111/j.0309-1317.2004.00519.x>

KEAR, Mark (2007). «Spaces of transition spaces of tomorrow: Making a sustainable future in Southeast False Creek, Vancouver». Cities [en línea], 24 (4), 324-334. <http://dx.doi.org/10.1016/j.cities.2007.01.005>

KeIl, Roger y Boudreau, Julie A. (2006). «Metropolitics and metabolics: Rolling out environmentalism in Toronto». En: HeYnen, Nik; Kaika, Maria y Swyngedouw, Erik (eds.). In the nature of cities: Urban political ecology and the politics of urban metabolism [en línea]. Londres: Routledge, 40-61.

Kenis, Anneleen y Lievens, Matthias (2015). The limits of the green economy: From re-inventing capitalism to re-politicising the present [en línea], 1-170. <http://dx.doi.org/10.4324/9781315769707> 
Kitchen, Lawrence (2013). "Are trees always “good”?: Urban political ecology and environmental justice in the valleys of South Wales». International Journal of Urban and Regional Research [en línea], 37 (6), 1968-1983. <http://dx.doi.org/10.1111/j.1468-2427.2012.01138.x>

March, Hug y Ribera-Fumaz, Ramón (2014a). «Smart contradictions: The politics of making Barcelona a Self-sufficient city». European Urban and Regional Studies [en línea], noviembre, 1-15. <http://dx.doi.org/10.1177/0969776414554488>

- (2014b). «Una revisión crítica desde la Ecología Política Urbana del concepto Smart City en el Estado español». Ecología Política, 47, 29-36.

Mclain, Rebecca J.; Hurley, Patrick T.; Emery, Marla R. y Poe, Melissa R. (2014). "Gathering "wild" food in the city: Rethinking the role of foraging in urban ecosystem planning and management». Local Environment [en línea], 19 (2), 220-240. <http://dx.doi.org/10.1080/13549839.2013.841659>

Milbourne, Paul (2012). «Everyday (in)justices and ordinary environmentalisms: Community gardening in disadvantaged urban neighbourhoods». Local Environment [en línea], 17 (9), 943-957. <http://dx.doi.org/10.1080/13549839.2011.607158>

Millington, Nate (2013). «Post-Industrial Imaginaries: Nature, Representation and Ruin in Detroit, Michigan». International Journal of Urban and Regional Research [en línea], 37 (1), 279-296. <http://dx.doi.org/10.1111/j.1468-2427.2012.01206.x>

MonstadT, Jochen (2009). «Conceptualizing the political ecology of urban infrastructures: Insights from technology and urban studies». Environmment and Planning $A$ [en línea], 41, 1924-1942. <http://dx.doi.org/10.1068/a4145>

Moore, Sarah (2006). «Forgotten roots of the Green City: Subsistence gardening in Columbus, Ohio, 1900-1940». Urban Geography [en línea], 27 (2), 174-192. <http://dx.doi.org/10.2747/0272-3638.27.2.174>

Morote, Álvaro F. y Hernández, María (2014). "Jardines y urbanizaciones: Nuevas naturalezas urbanas en el litoral de la provincia de Alicante». Documents d'Anàlisi Geogràfica [en línea], 60 (3), 483-504. <http://dx.doi.org/10.5565/rev/dag.122>

NJeru, Jeremia (2010). "Defying" democratization and environmental protection in Kenya: The case of Karura Forest reserve in Nairobi». Political Geography [en línea], 29 (6), 333-342. <http://dx.doi.org/10.1016/j.polgeo.2010.07.003>

- (2013). "Donor-driven" neoliberal reform processes and urban environmental change in Kenya: The case of Karura Forest in Nairobi». Progress in Development Studies [en línea], 13 (1), 63-78.

<http://dx.doi.org/10.1177/146499341201300105>

Padullés, Josep; Vila, Josep y Barriocanal, Carles (2014a). «Maintenance, Modifications, and Water Use in Private Gardens of Alt Empordà, Spain». HortTechnology, 24 (3), 374-383.

- (2014b). «Examining floristic boundaries between garden types at the global scale». Investigaciones Geográficas [en línea], 61 (1), 71-86. <http://dx.doi.org/10.14198/ingeo2014.61.05> 
PARÉs, Marc (2005). «Espai públic enjardinat: Impactes ambientals, model urbà i individualització a la Regió Metropolitana de Barcelona». Documents d'Anàlisi Geogràfica, 45, 91-109.

Parés, Marc; Domene, Elena y Saurí, David (2004). «Gestión del agua en la jardinería pública y privada de la Región Metropolitana de Barcelona». Boletín de la Asociación de Geógrafos Españoles, 37, 223-237.

Parés, Marc; March, Hug y Saurí, David (2013). "Atlantic Gardens in Mediterranean Climates: Understanding the Production of Suburban Natures in Barcelona». International Journal of Urban and Regional Research [en línea], 37 (1), 328-347. <http://dx.doi.org/10.1111/j.1468-2427.2012.01118.x>

Perkins, Harold A.; Heynen, Nik y Wilson, Joe (2004). «Inequitable access to urban reforestation: The impact of urban political economy on housing tenure and urban forests». Cities [en línea], 21 (4), 291-299. $<$ http://dx.doi.org/10.1016/j.cities.2004.04.002>

Quastel, Noah; Moos, Markus y Lynch, Nicholas (2012). «Sustainability-as-density and the return of the social: The case of Vancouver, British Columbia». Urban Geography [en línea], 33 (7), 1055-1084. <http://dx.doi.org/10.2747/0272-3638.33.7.1055>

Robbins, Paul (2007). Lawn People: How Grasses, Weeds, and Chemicals Make Us Who We Are. Filadelfia: Temple University Press.

Robbins, Paul y Birkenholtz, Trevor (2003). "Turf-grass revolution: Measuring the expansion of the American lawn». Land Use Policy [en línea], 20 (2), 181-194. <http://dx.doi.org/10.1016/s0264-8377(03)00006-1>

Robbins, Paul; Polderman, Anne M. y Birkenholtz, Trevor (2001). «Lawns and Toxins: An Ecology of the City». Cities [en línea], 18 (6), 369-380. <http://dx.doi.org/10.1016/s0264-2751(01)00029-4>

Robbins, Paul y Sharp, Julie (2003). "The Lawn-Chemical Economy and its Discontents». Antipode [en línea], 35 (5), 955-979. <http://dx.doi.org/10.1111/j.1467-8330.2003.00366.x>

- (2006). «Turfgrass subjects: The political economy of urban monoculture». En: Heynen, Nik; Kaika, Maria y Swyngedouw, Erik (eds.). In the nature of cities: Urban political ecology and the politics of urban metabolism. Londres: Routledge, 106-123.

- (2008). «Producing and consuming chemicals: The moral economy of the american lawn». En: Marzluff, John M. et al. (eds.). Urban Ecology: An International Perspective on the Interaction between Humans and Nature. Nueva York: Springer, 181-205.

SAFrANSKY, Sara (2014). "Greening the urban frontier: Race, property, and resettlement in Detroit». Geoforum [en línea], 237-248. <http://dx.doi.org/10.1016/j.geoforum.2014.06.003>

Shillington, Laura J. (2013). «Right to food, right to the city: Household urban agriculture, and socionatural metabolism in Managua, Nicaragua». Geoforum [en línea], 44, 103-111. <http://dx.doi.org/10.1016/j.geoforum.2012.02.006>

Troy, Laurence (2014). «(Re)Producing nature in Pyrmont and Ultimo». Geographical Research [en línea], 52 (4), 387-399. <http://dx.doi.org/10.1111/1745-5871.12074> 


\section{Flujos metabólicos}

Anguelovski, Isabelle (2015). «Alternative food provision conflicts in cities: Contesting food privilege, injustice, and whiteness in Jamaica Plain, Boston». Geoforum [en línea], 58, 184-194. <http://dx.doi.org/10.1016/j.geoforum.2014.10.014>

Biehler, Dawn D. (2009). "Permeable homes: A historical political ecology of insects and pesticides in US public housing». Geoforum [en línea], 40 (6), 1014-1023. <http://dx.doi.org/10.1016/j.geoforum.2009.08.004>

BJerkli, Camilla Louise (2015). «Power in waste: Conflicting agendas in planning for integrated solid waste management in Addis Ababa, Ethiopia». Norsk Geografisk Tidsskrift [en línea], 69 (1), 18-27. <http://dx.doi.org/10.1080/00291951.2014.992806>

Bruzzelli, Michael (2008). "A political ecology of scale in urban air pollution monitoring». Transactions of the Institute of British Geographers [en línea], 33 (4), 502517. <http://dx.doi.org/10.1111/j.1475-5661.2008.00316.x>

Demaria, Federico y Schindler, Seth (2015). «Contesting Urban Metabolism: Struggles Over Waste-to-Energy in Delhi, India». Antipode [en línea], 1-21. <http://dx.doi.org/10.1111/anti.12191>

Graham, Stephen (2015). «Life support: The political ecology of urban air». City [en línea], 19 (2-3), 192-215. <http://dx.doi.org/10.1080/13604813.2015.1014710>

Heynen, Nik (2006b). "Justice of eating in the city: the political ecology of urban hunger». En: Heynen, Nik; KaIka, Maria y Swyngedouw, Erik (eds.): In the nature of cities: Urban political ecology and the politics of urban metabolism. Londres: Routledge, 124-136.

Heynen, Nik; Perkins, Harold A. y Roy, Parama (2006b). «The political ecology of uneven urban green space: The impact of political economy on race and ethnicity in producing environmental inequality in Milwaukee». Urban Affairs Review [en línea], 42 (1), 3-25. <http://dx.doi.org/10.1177/1078087406290729>

Holifield, Ryan (2009). "Actor-Network theory as a critical approach to environmental justice: A case against synthesis with urban political ecology». Antipode [en línea], 41 (4), 637-658. <http://dx.doi.org/10.1111/j.1467-8330.2009.00692.x>

Houston, Donna y Ruming, Kristian (2014). «Suburban toxicity: A political ecology of asbestos in Australian cities». Geographical Research [en línea], 52 (4), 400-410. <http://dx.doi.org/10.1111/1745-5871.12075>

Instone, Lesley y SweEney, Jill (2014). «Dog waste, wasted dogs: The contribution of human-dog relations to the political ecology of Australian urban space». Geographical Research [en línea], 52 (4), 355-364. <http://dx.doi.org/10.1111/1745-5871.12059>

Kay, Samuel; Zhao, Bo y Sui, Daniel (2015). "Can Social Media Clear the Air?: A Case Study of the Air Pollution Problem in Chinese Cities». Professional Geographer [en línea], 67 (3), 351-363. <http://dx.doi.org/10.1080/00330124.2014.970838>

Lawhon, Mary (2013). «Flows, friction and the sociomaterial metabolization of alcohol». Antipode [en línea], 45 (3), 681-701. <http://dx.doi.org/10.1111/j.1467-8330.2012.01028.x> 
LEONARD, Llewellyn (2012). «Another political ecology of civil society reflexiveness against urban industrial risks for environmental justice: The case of the Bisasar landfill, Durban, South Africa». Singapore Journal of Tropical Geography [en línea], 33 (1), 77-92.

<http://dx.doi.org/10.1111/j.1467-9493.2012.00448.x>

Marvin, Simon y MedD, Will (2006). «Metabolism of obe-city: Flows of fat through bodies, cities and sewers». En: Heynen, Nik; Kaika, Maria y Swyngedouw, Erik (eds.). In the nature of cities: Urban political ecology and the politics of urban metabolism. Londres: Routledge, 137-149.

McClintock, Nathan (2015). «A critical physical geography of urban soil contamination». Geoforum [en línea], 65, 69-85. <http://dx.doi.org/10.1016/j.geoforum.2015.07.010>

Moore, Sarah A. (2008). "The politics of garbage in Oaxaca, Mexico». Society and Natural Resources [en línea], 21 (7), 597-610. <http://dx.doi.org/10.1080/08941920701759551>

Moragues-Faus, Ana y Morgan, Kevin (2015). "Reframing the foodscape: The emergent world of urban food policy». Environment and Planning $A$ [en línea], 47 (7), 1558-1573. <http://dx.doi.org/10.1177/0308518X15595754>

Morgan, Kevin (2015). "Nourishing the city: The rise of the urban food question in the Global North». Urban Studies [en línea], 52 (8), 1379-1394. <http://dx.doi.org/10.1177/0042098014534902>

Mulligan, Kate; Elliott, Susan J. y Schuster-Wallace, Corinne J. (2012). «The place of health and the health of place: Dengue fever and urban governance in Putrajaya, Malaysia». Health and Place [en línea], 18 (3), 613-620. <http://dx.doi.org/10.1016/j.healthplace.2012.01.001>

NJeru, Jeremia (2006). "The urban political ecology of plastic bag waste problem in Nairobi, Kenya». Geoforum [en línea], 37 (6), 1046-1058. <http://dx.doi.org/10.1016/j.geoforum.2006.03.003>.

Ostos, Joan Ramon (2014). «Erik Swyngedouw y la ecología política urbana». Ecología Política, 47, 110-118.

Parizeau, Kate (2015). "Urban political ecologies of informal recyclers' health in Buenos Aires, Argentina». Health and Place [en línea], 33, 67-74. <http://dx.doi.org/10.1016/j.healthplace.2015.02.007>

Perkins, Harold A. (2007). «Ecologies of actor-networks and (non)social labor within the urban political economies of nature». Geoforum [en línea], 38 (6), 1152-1162. <http://dx.doi.org/10.1016/j.geoforum.2007.01.007>

SAGUIN, Kristian (2014). «Biographies of fish for the city: Urban metabolism of Laguna Lake aquaculture». Geoforum [en línea], 54, 28-38. <http://dx.doi.org/10.1016/j.geoforum.2014.03.008>

Solíz, María F. (2014). "Ecología política y geografía crítica de la basura en el Ecuador: Determinación social y conflictos distributivos». Ecología Politica, 47, 56-61.

VÉRON, René (2006). "Remaking urban environments: The political ecology of air pollution in Delhi». Environment and Planning A [en línea], 38 (11), 2093-2109. <http://dx.doi.org/10.1068/a37449>

Yates Julian, S. y Gutberlet, Jutta (2011). «Reclaiming and recirculating urban natures: Integrated organic waste management in Diadema, Brazil». Environment and Planning $A$ [en línea], 43 (9), 2109-2124. $<$ http://dx.doi.org/10.1068/a4439> 


\section{Ecología politica urbana de los riesgos naturales}

Ajibade, Idowu y McBean, Gordon (2014). "Climate extremes and housing rights: A political ecology of impacts, early warning and adaptation constraints in Lagos slum communities». Geoforum [en línea], 55, 76-86.

<http://dx.doi.org/10.1016/j.geoforum.2014.05.005>

BAXter, Vern (2014). «Rent, Real Estate, and Flood Mitigation in New Orleans East». Antipode, 46 (4), 1014-1031.

<http://dx.doi.org/10.1111/anti.12080>

Collins, Timothy W. (2010). «Marginalization, facilitation, and the production of unequal risk: The 2006 Paso del Norte floods». Antipode [en línea], 42 (2), 258-288. <http://dx.doi.org/10.1111/j.1467-8330.2009.00755.x>

Giglioli, Ilaria y Swyngedouw, Erik (2008). «Let's Drink to the Great Thirst!: Water and the Politics of Fractured Techno-natures in Sicily». International Journal of Urban and Regional Research [en línea], 32 (2), 392-414.

<http://dx.doi.org/10.1111/j.1468-2427.2008.00789.x>

Gustafson, Seth (2015a). "Maps and contradictions: Urban political ecology and cartographic expertise in southern Appalachia». Geoforum [en línea], 60, 143-152. <http://dx.doi.org/10.1016/j.geoforum.2015.01.017>

- (2015b). "The making of a landslide: Legibility and expertise in exurban southern Appalachia». Environment and Planning A [en línea], 47 (7), 1404-1421. <http://dx.doi.org/10.1177/0308518X15595767>

Ioris, Antonio A.R. (2012c). "The geography of multiple scarcities: Urban development and water problems in Lima, Peru». Geoforum [en línea], 43 (3), 612-622. <http://dx.doi.org/10.1016/j.geoforum.2011.12.005>

KaIKA, Maria (2003). "Constructing Scarcity and Sensationalising Water Politics: 170 Days that Shook Athens». Antipode [en línea], 35 (5), 919-953. <http://dx.doi.org/10.1111/j.1467-8330.2003.00365.x>

- (2005). City of Flows: Modernity, Nature and the City. Londres: Routledge.

- (2006). "The political ecology of water scarcity: The 1989-1991 Athenian drought». En: Heynen, Nik; Kaika, Maria y Swyngedouw, Erik (eds.). In the nature of cities: Urban political ecology and the politics of urban metabolism. Londres: Routledge [en línea], 150-164. <http://dx.doi.org/10.2747/0272-3638.28.2.206>

Marks, Danny (2015). «The urban political ecology of the 2011 floods in Bangkok: The creation of uneven vulnerabilities». Pacific Affairs [en línea], 88 (3), 623-651. <http://dx.doi.org/10.5509/2015883623>

Mustafa, Daanish (2005). "The production of an urban hazardscape in Pakistan: Modernity, vulnerability, and the range of choice». Annals of the Association of American Geographers [en línea], 95 (3), 566-586. <http://dx.doi.org/10.1111/j.1467-8306.2005.00475.x>

Otero, Iago; Kallis, Giorgos; Aguilar, Raúl y Ruiz, Vicenç (2011). «Water scarcity, social power and the production of an elite suburb: The political ecology of water in Matadepera, Catalonia». Ecological Economics [en línea], 70 (7), 1297-1308. <http://dx.doi.org/10.1016/j.ecolecon.2009.09.011>

Padawangi, Rita y Douglass, Mike (2015). «Water, water everywhere: Toward participatory solutions to chronic urban flooding in Jakarta». Pacific Affairs [en línea], 88 (3), 517-550.

<http://dx.doi.org/10.5509/2015883517> 
Pelling, Mark (1999). "The political ecology of flood hazard in urban Guyana». Geoforum [en línea], 30 (3), 249-261. <http://dx.doi.org/10.1016/s0016-7185(99)00015-9>

Ranganathan, Malini (2015). «Storm Drains as Assemblages: The Political Ecology of Flood Risk in Post-Colonial Bangalore». Antipode [en línea], 47 (5), 1300-1320. $<$ http://dx.doi.org/10.1111/anti.12149>

Romero, Hugo; Fuentes, Claudio y Smith, Pamela (2010). «Ecología política de los riesgos naturales y de la contaminación ambiental en Santiago de Chile: Necesidad de justicia ambiental». Scripta Nova: Revista Electrónica de Geografía y Ciencias Sociales [en línea], XIV (331), 52. Barcelona: Universidad de Barcelona. <http:// www.ub.edu/geocrit/sn/sn-331/sn-331-52.htm>.

Simon, Gregory L. (2014). «Vulnerability-in-Production: A Spatial History of Nature, Affluence, and Fire in Oakland, California». Annals of the Association of American Geographers [en línea], 104 (6), 1199-1221. <http://dx.doi.org/10.1080/00045608.2014.941736>

Simon, Gregory L. y Dooling, Sarah (2013). "Flame and fortune in California: The material and political dimensions of vulnerability». Global Environmental Change [en línea], 23 (6), 1410-1423.

<http://dx.doi.org/10.1016/j.gloenvcha.2013.08.008> 